\title{
The Detection of BANA Hydrolysis Activity in Abutment Teeth in Patients with Removable Partial Denture
}

\author{
1Jigar P Kalaria, ${ }^{2}$ Birood G Patel, ${ }^{3}$ Vilas Patel, ${ }^{4}$ Charu Agrawal
}

\begin{abstract}
Purpose: The aim of this study was to detect the presence of N-benzyl-Dl-arginase-2-naphthalamide (BANA) microorganisms in chronic periodontitis of abutment teeth in subjects wearing removable partial dentures (RPDs) by measuring the red complex using BANA-Zyme ${ }^{\mathrm{TM}}$ test.

Materials and methods: Twenty-five subjects (mean age: 59.3 years) with Kennedy class II partially edentulous arch participated in this study. The microbiological record of red complex was done by BANA-Zyme kit and clinical parameters, such as plaque index (PI), gingival index ( $\mathrm{GI}$ ) and probing depth were recorded at abutment teeth in contact with the direct retainer of unilaterally designed RPDs and the corresponding nonabutment teeth on the opposite side.
\end{abstract}

Results: Mean scores of BANA for the red complex, PI and GI of the abutment teeth were significantly greater than those of the nonabutment teeth $(p<0.01)$. No significant mean difference in pocket depth (PD) was found between the abutment and nonabutment teeth. Logistic regression analysis revealed that abutment teeth, high scores of $\mathrm{PI}$ and a maintenance interval longer than 6 months were significant predictors for positive red complex scores $(p<0.05)$.

Conclusion: These results suggest that the microbiological risk for periodontitis of abutment teeth is greater than that for nonabutment teeth in RPD wearers.

Keywords: BANA-Zyme ${ }^{\mathrm{TM}}$ test, Microbiological risk, Periodontitis, Red complex, Removable partial denture.

How to cite this article: Kalaria JP, Patel BG, Patel V, Agrawal C. The Detection of BANA Hydrolysis Activity in Abutment Teeth in Patients with Removable Partial Denture. Int J Prosthodont Restor Dent 2015;5(2):31-37

Source of support: Nil

Conflict of interest: None

\footnotetext{
1,2Postgraduate Student (3rd year), ${ }^{3,4}$ Reader

${ }^{1,4}$ Department of Periodontics, Narsinhbhai Patel Dental College, Visnagar, Gujarat, India

${ }^{2,3}$ Department of Prosthodontics and Crown and Bridge Narsinhbhai Patel Dental College, Visnagar, Gujarat, India
}

Corresponding Author: Jigar P Kalaria, Postgraduate Student (3rd year), Department of Periodontics, Narsinhbhai Patel Dental College, Visnagar-384315, Gujarat, India, Phone: 09427724846, e-mail: jigarkalaria@gmail.com

\section{INTRODUCTION}

Periodontal diseases, now recognized as bacterial infections, are among the most common, chronic diseases of humans, affecting 5 to $30 \%$ the adult population in the age group of 25 to more than 75 years.

Several methods, such as cultural, microscopic examination, immunologic reagents, enzyme activity, and deoxyribonucleic acid (DNA) probes, could help the clinician monitor plaques for the presence of periodontal pathogens in conjunction with clinical parameters.

Evidence has been presented which suggests a specific bacterial etiology in many forms of periodontal diseases. The association of a limited number of bacterial species, i.e. Haemophilus (Actinobacillus) actinomycetemcomitans, Porphyromonas gingivalis, $T(B)$ forsythia, Wolinella recta and spirochetes with various forms of periodontal disease allows for the development of diagnostic tools that are based upon the detection of one or more of these in plaque samples. ${ }^{1}$

The N-benzyl-Dl-arginase-2-naphthalamide (BANA) test offers the potential to identify plaques that may harbor Treponema denticola, $P$. gingivalis and a third organism, T. forsythus, which has recently been associated with the progressing periodontal lesion, The BANA test would then be a simple method of detecting periodontal anaerobic infections. ${ }^{2}$

The BANA test is quite effective for the detection of red complex organisms and is useful in the initial diagnosis of chronic periodontitis. Loesche proposed the use of BANA reaction to detect the presence of periodontal pathogens, and thus serve as a marker of disease activity and also aid in monitoring periodontal therapy. ${ }^{3}$

The BANA test has been used in several studies. The results of the BANA test have been previously compared with other microbiological techniques, such as enzymelinked immunoabsorbent assay (ELISA) reaction ${ }^{4}$ and polymerize chain reaction (PCR). ${ }^{5}$ However, these studies compared only the bacterial enzymatic activity with the presence or absence of the microorganisms. Additionally, it is not clear whether a BANA-positive reaction is associated with isolated or simultaneous presence of the red complex species. ${ }^{6}$

The investigation demonstrated that the ability of subgingival plaque to hydrolyze BANA was significantly associated with (1) elevated numbers of spirochetes present 
in those plaque samples and (2) the clinical diagnosis of the sampled sites, i.e. diseased sites in untreated patients and unresponsive sites in treated patients. Conversely, healthy and responsive sites were almost universally associated with BANA-negative reactions and harbored low numbers of spirochetes.

Removable partial denture (RPD) is a treatment option for partially edentulous patients. The preservation of the remaining teeth and periodontal tissue in a state of health is one of the objectives for RPD. Oral hygiene is the important for prevent the caries, gingivitis and periodontitis, on abutment teeth in case of RPD. ${ }^{8-10}$

There are many studies demonstrated that wearing of RPDs could increase accumulation of dental plaque ${ }^{11,12}$ due to the coverage of marginal gingival tissue with RPDs enhanced accumulation of dental plaque and gingival inflammation of abutment teeth. ${ }^{13,14}$

Because of this, maintenance of oral hygiene and proper plaque control is much important to prevent adverse effect of deposits. And it is very important to identify the bacteria to prevent disease progression.

So, the BANA test has been successfully used in several clinical trials to evaluate changes in the subgingival microbiota after therapy. ${ }^{15}$

So, from the above facts, it was found that the BANA test kit is helpful in providing a rationale for implementing treatment, and allows the operator to intercept the disease at a primary level and also indirectly enable the clinician to monitor sites for the development of active periodontal disease and even predict future attachment loss.

The aim of this study was to detect the presence of BANA microorganisms in chronic periodontitis of abutment teeth in subjects wearing removable partial dentures (RPDs) by measuring the red complex using BANA-Zyme ${ }^{\mathrm{TM}}$ (oratec corporation manassas, virginia, USA) test.

\section{MATERIALS AND METHODS}

\section{Subjects}

Twenty-five subjects participated in this study. The subjects were recruited from patients seeking treatment at the Narsinhbhai Patel Dental College and Hospital. Patients who had a unilateral distal edentulous space extending from the second premolar or first molar to third molar and had them replaced with unilaterally designed RPDs were included in the study. According to a previous study, ${ }^{16}$ patients who had received periodontal therapy or antibiotic therapy in the last 3 months were excluded from the study. Each subject received a written and oral description of the experimental procedures, and informed consent was obtained prior to enrollment in the study.

Each subject had a single RPD in the maxilla or mandible. Wearing period of RPDs (denture age) ranged from 14 to 54 months (mean: 31.2 months). All subjects had acrylic resin RPDs. The acrylic resin RPDs had a backaction clasp with a mesio-occlusal rest as direct retainer and an embrasure hook as indirect retainer. The marginal gingival tissue on the lingual side of abutment teeth was covered by the major connector in acrylic resin RPDs. A great majority of the subjects (92\%: $23 / 25$ ) performed tooth cleaning two or more times daily. The subjects were receiving oral maintenance care at Narsinhbhai Patel Dental College and Hospital. The interval from the last oral maintenance visit to the examination ranged from 5 to 25 months.

\section{Examined Teeth}

In each subject, an abutment tooth in contact with the direct retainer of RPD and the corresponding tooth on the opposite side of the same arch were selected for examination. Twenty-five second premolars on the edentulous side in the maxilla or mandible, 50 teeth in total, were examined by a single examiner. None of the examined teeth were abutments of a fixed partial denture or connected with adjunct teeth.

\section{Microbiological Examination}

Microbiological examination was performed to assess the red complex using a BANA-Zyme ${ }^{\mathrm{TM}}$ test $^{17}$ (Fig. 1). Supragingival plaque on the examined teeth was removed using cotton swab to isolate the examined area prior to sampling. Subgingival plaque was collected using a probe in one pocket at distal side of abutment and nonabutment teeth for each subject. Curette was not used to collect sample because contamination of blood with collected plaque affects the results of BANA test. A test strip of BANA test has two reagent strips on the surface. After the subgingival plaque specimens were collected and placed onto the lower reagent strip (Fig. 2), the upper reagent strip was moistened with distilled water using a cotton swab (Fig. 3). The reagent upper strip was folded (Fig. 4) in order to contact the lower reagent, and it was inserted into a heating block and incubated for 5 minutes at $55^{\circ} \mathrm{C}$ (Fig. 5). Naphthylamide liberated from the lower reagent strip diffused into the upper reagent strip where it reacted with a dye to form a permanent blue color. Scores were given based on the color of the strip by visual inspection (Fig. 6 and Table 1).

When BANA test showed no blue color (score 0), it indicated that number of bacteria was less than clinically significant level in all three species of red complex.

When BANA test showed faint blue color (score 1), number of bacteria was more than clinically significant level at least in one species of red complex, but less than high level. 


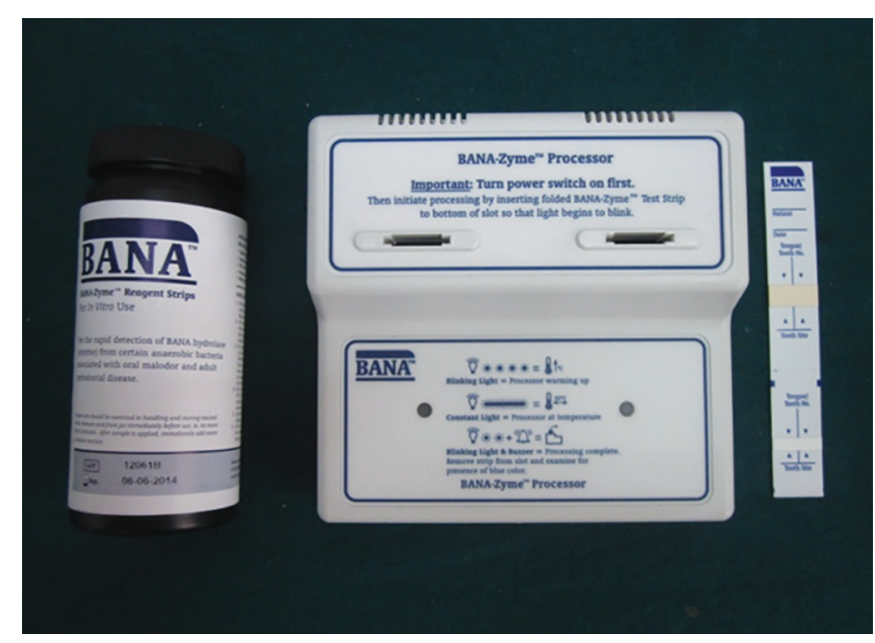

Fig. 1: N-benzyl-Dl-arginase-2-naphthalamide Zyme ${ }^{\mathrm{TM}}$ Kit

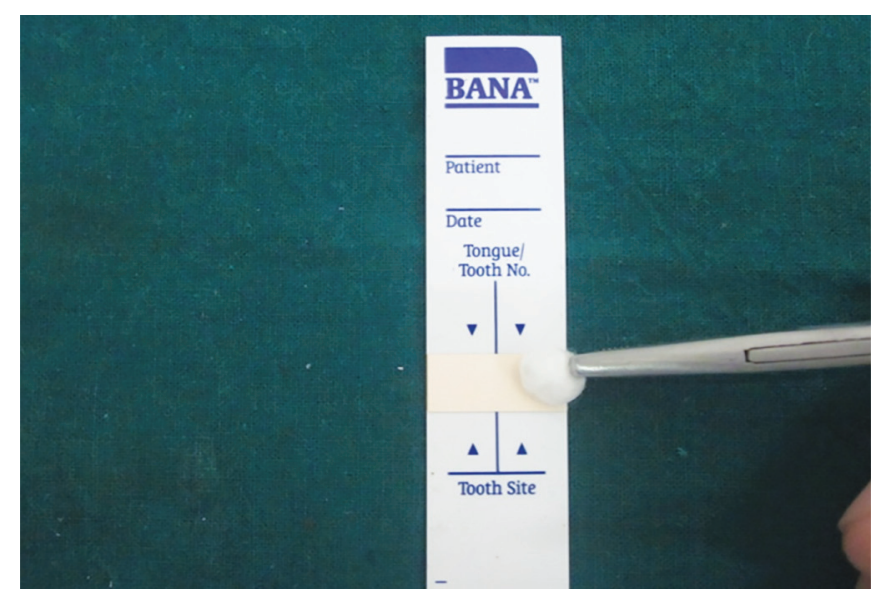

Fig. 3: Upper part of strip is wet with distilled water by cotton swab

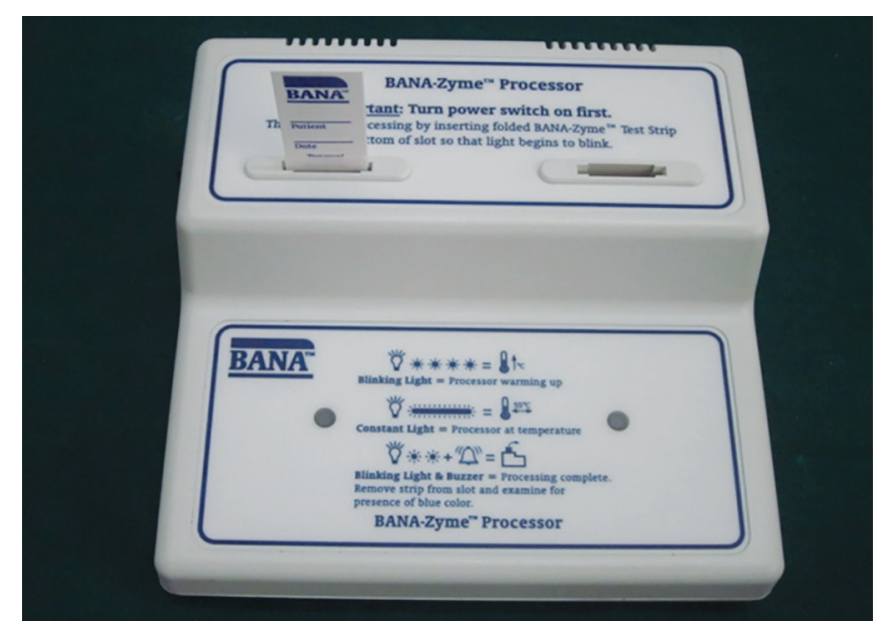

Fig. 5: Incubation in processor by putting the strip in the processor

When BANA test showed distinct blue (score 2), number of bacteria was more than the increased level at least in one species of red complex.

Score 1 and 2 represented that red complex was present clinically at the examined site. BANA-Zyme ${ }^{\mathrm{TM}}$ test is simple to perform and can provide results within 15 minutes atchairside. In a previous study, BANA-Zyme ${ }^{\mathrm{TM}}$

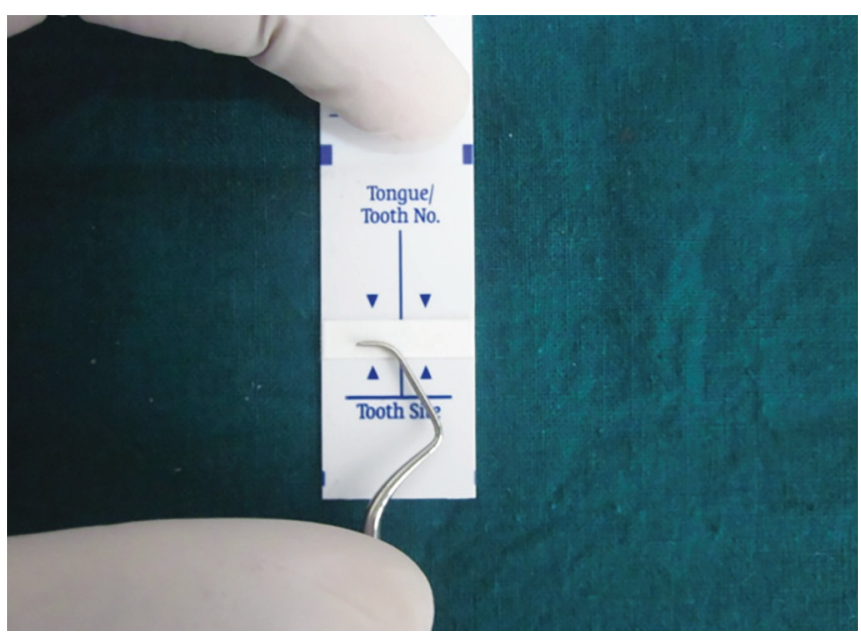

Fig. 2: Sample placement on BANA strip

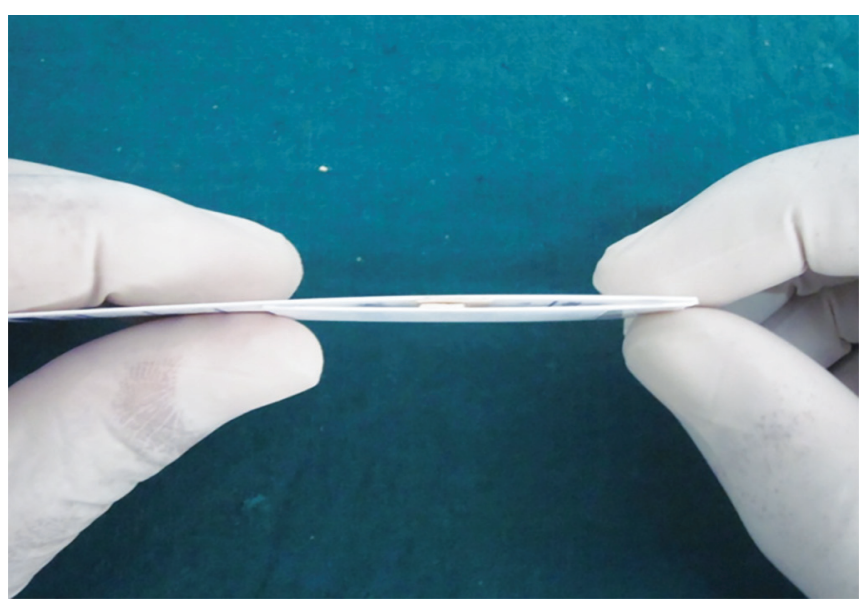

Fig. 4: Fold the strip

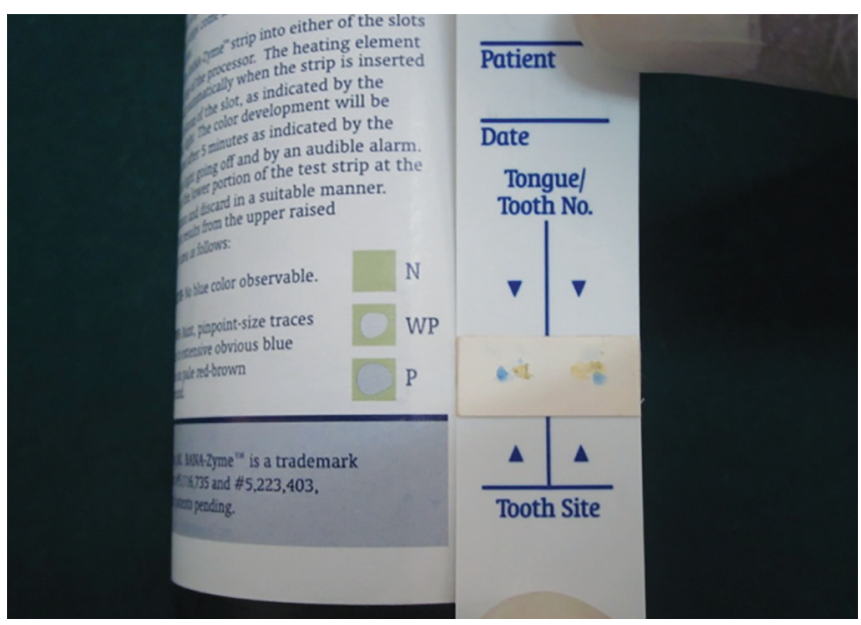

Fig. 6: Result analysis by visual inspection

test, the DNA probes and enzyme-linked immunosorbent assay procedure exhibited high sensitivity (90-96\%) and high accuracy $(83-92 \%) .{ }^{17}$

In our preliminary experiment, test-retest of BANAZyme $^{\mathrm{TM}}$ test within a 2-week interval was performed by a single examiner in 25 abutment teeth in 25 patients with RPDs. The Kappa value was 0.72 , indicating that this test was clinically acceptable. 
Table 1: Interpretation of score for BANA-Zyme ${ }^{\mathrm{TM}}$ test

\begin{tabular}{lll}
\hline Score & Number of bacteria & Color identified \\
\hline 0 & P. gingivalis $<1.0 \times 10^{6}$ and & No blue color \\
& T. denticola $<2.2 \times 10^{6}$ and & \\
& T. forsythia $<1.0 \times 10^{6}$ & \\
1 & P. gingivalis $1.2 \times 10^{6}-4.4 \times 10^{6}$ or & Faint blue color \\
& T. denticola $2.7 \times 10^{6}-1.1 \times 10^{6}$ or & \\
T. forsythia $1.0 \times 10^{6}-1.0 \times 10^{6}$ & \\
2 & P. gingivalis $>5.9 \times 106$ or & Distinct blue \\
T. denticola $>1.6 \times 107$ or & color \\
T. forsythia $>1.0 \times 107$ & \\
\hline
\end{tabular}

\section{Clinical Examination}

Clinical examination was performed to assess oral hygiene, gingival inflammation and PD. Plaque index $(\mathrm{PI})$, gingival index $(\mathrm{GI})$ and pocket depth $(\mathrm{PD})$ were evaluated at distal side of the abutment and nonabutment teeth. Plaque index was evaluated before BANA test. Gingival index and PD was evaluated after BANA test. The oral hygiene was assessed based on PI according to criteria by Silness and Loe. ${ }^{18}$ Gingival inflammation was assessed as GI according to criteria by Silness and Loe. ${ }^{19}$ Probing PD was measured as the distance from the gingival margin to the bottom of the clinical pocket using a williams probe inserted with gentle pressure.

\section{STATISTICAL ANALYSIS}

The null hypothesis to be tested in this study would be that the red complex was detected in equal amounts on abutment teeth and nonabutment teeth. Difference in mean scores of red complex, PI, PD and GI between abutment teeth and nonabutment teeth were tested using Wilcoxon's signed rank sum test. A logistic regression analysis was performed using red complex as an outcome, and abutment/nonabutment tooth, clinical parameters (PI, GI and PD), maintenance interval and denture age as predictors. In the regression analysis, 0 was given for score 0 in red complex and 1 for score 1 or 2 , and a forward stepwise method was used for the selection of predictors. A p-value less than 0.05 was considered significant. Statistical package for the social sciences (SPSS) $16.0 \mathrm{v}$ was used for statistical analyses.

\section{RESULTS}

Figure 1 shows the percentage distribution of scores for the red complex, PI and GI on nonabutment and abutment teeth. Higher scores were found more frequently in abutment teeth compared with nonabutment teeth.

For example, $22(44 \%)$ of 50 abutment teeth and 10 $(20 \%)$ of 50 nonabutment teeth showed score 2 for red complex (Graph 1). 18 (36\%) of 50 abutment teeth and $8(16 \%)$ of 50 nonabutment teeth showed score 2 for PI (Graph 2). 25 (50\%) of 50 abutment teeth and 18 (36\%) of 50 nonabutment teeth showed score 2 or 3 for GI. (Graph 3).

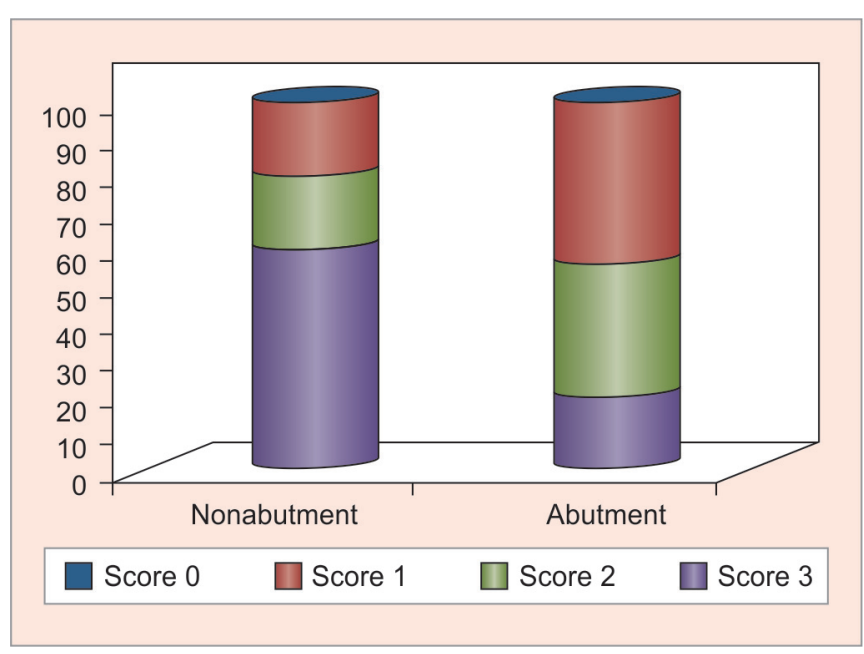

Graph 1: Percentage distribution of scores for red complex of nonabutment teeth (Nonabut, $\mathrm{n}=25$ ) and abutment teeth (Abut, $\mathrm{n}=25)$

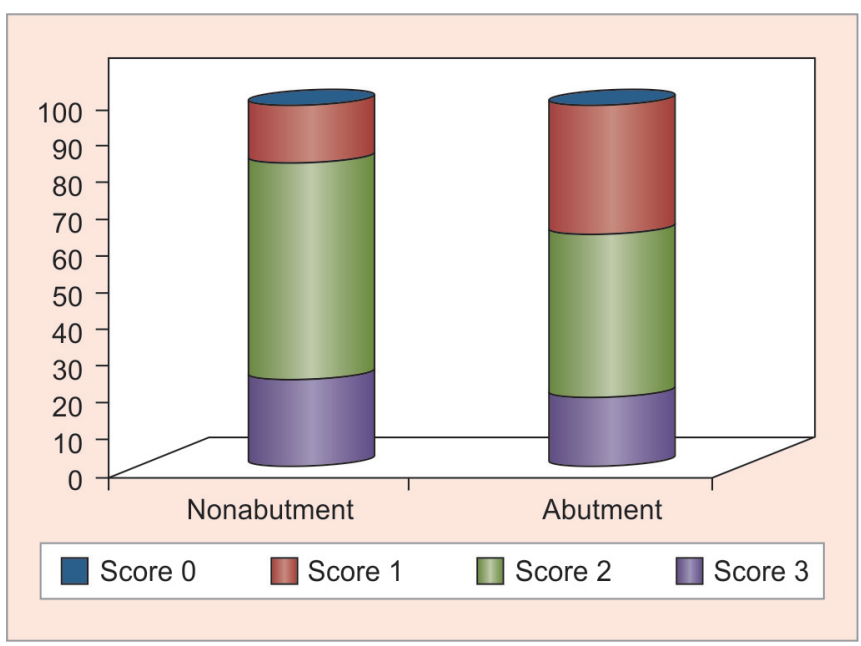

Graph 2: Percentage distribution of scores for plaque index of nonabutment teeth (Nonabut, $\mathrm{n}=25$ ) and abutment teeth (Abut, $\mathrm{n}=25)$

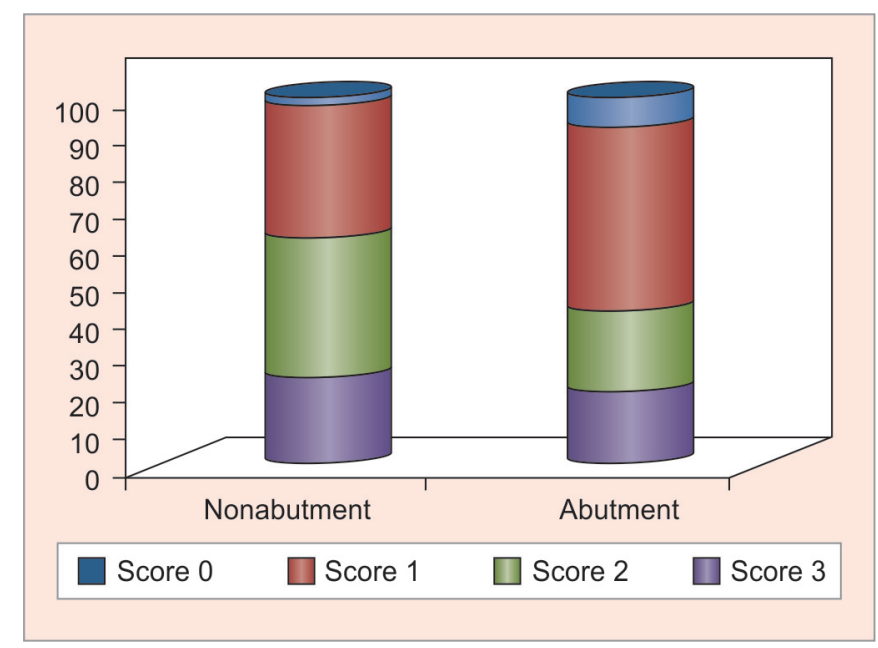

Graph 3: Percentage distribution of scores for gingival index of nonabutment teeth (Nonabut, $\mathrm{n}=25$ ) and abutment teeth (Abut, $\mathrm{n}=25)$ 
Table 2: Mean scores and SD of red complex, PI, GI and PD on nonabutment teeth $(n=25)$ and abutment teeth $(n=25)$

\begin{tabular}{lllll}
\hline Parameter & Tooth & Mean & $S D$ & $p$ \\
\hline Red complex & Nonabutment & 0.60 & 0.81 & 0.001 \\
(BANA) & Abutment & 1.00 & 0.64 & \\
Plaque index & Nonabutment & 0.76 & 0.49 & 0.002 \\
& Abutment & 1.20 & 0.37 & \\
Gingival index & Nonabutment & 1.12 & 0.52 & 0.002 \\
& Abutment & 1.42 & 0.88 & \\
Pocket depth (mm) & Nonabutment & 2.31 & 0.56 & 0.071 \\
& Abutment & 2.43 & 0.71 & \\
\hline
\end{tabular}

Table 2 shows the mean scores for red complex, PI, GI and PD on nonabutment teeth and abutment teeth. The mean scores for red complex, PI and GI on the abutment teeth were significantly greater than for the nonabutment teeth $(\mathrm{p}<0.01)$. The PD at the distal side of all examined teeth were less than $4 \mathrm{~mm}$, and no significant mean difference was found between abutment and nonabutment teeth $(\mathrm{p}=0.07)$. The regression analysis found that abutment teeth, higher score of PI and a maintenance interval longer than 6 months were significant predictors for positive red complex scores $(p<0.05)$. Gingival index, PD and denture age were not significant predictors.

\section{DISCUSSION}

In this study, patients were receiving oral maintenance care after denture delivery at Narsinhbhai Patel Dental College and Hospital. The PD of examined teeth in this study was less than $4 \mathrm{~mm}$, suggesting that supporting tissues of the examined teeth had not deteriorated to the stage of periodontitis and there was no periodontal examination was performed before denture fabrication, this study could not provide any evidence of a causeeffect relationship. These limitations should be taken into consideration while interpreting the results of this study.

N-benzyl-Dl-arginase-2-naphthalamide test was employed to perform microbiological examination in this study. Subsequent studies implicated the cultivable spirochete, $T$. denticola and B. forsythus as also being involved in periodontal disease. ${ }^{8-10}$ Grossi et $\mathrm{al}^{20,21}$ in an epidemiological investigation involving over 1,300 adults, identified several risk factors for attachment and alveolar bone loss including the presence of subgingival P. gingivalis and B. forsythus, as well as smoking.

The BANA test is a simple, inexpensive chairside in vitro test which can be used in the dental office. The test is designed to detect the presence of one or more anaerobic bacteria commonly associated with periodontal disease, namely, T. denticola, P. gingivalis, and B. forsythus in plaque samples taken from periodontally diseased teeth. This information may help the clinician in the choice of an antimicrobial agent specific for anaerobes in the case of an individual who presents with advanced periodontal disease of the severity that usually would require surgical intervention.

The clinician might also use the presence of these BANA positive species to monitor the adequacy of treatment. The BANA test could alert the clinician to the present of a subclinical/clinical periodontal infection in smokers, or in individuals at risk to cardiovascular diseases.

Thus, the preponderance of evidence indicates that T. denticola, P. gingivalis, and B. forsythus are periodontal pathogens and because all are anaerobes, it would appear that periodontal disease is a chronic infection due to the overgrowth of certain anaerobic members of the plaque flora. This suggests that the BANA test can be used to detect in plaque samples the same three anaerobic species that have been associated with periodontal disease by investigators who have used DNA probes, cultural methods and immunological reagents.

The BANA test is based on a modification of the BANA hydrolysis test of Loesche et $\mathrm{al}^{3,22-24}$ which in turn was an adaptation of the trypsin-like enzyme contained in the API-ZYM kit. The anaerobic bacteria P. (Bacteroides) gingivalis, B. forsythus and/or T. denticola are unique in the subgingival flora in that they possess a trypsinlike enzyme which hydrolyzes the synthetic peptide N-benzyl-Dl-arginase-2-naphthalamide (BANA).,23,24

Socransky et al and Haffajee et al (1997) in their extensive study involving over 10,000 plaque samples taken from over 100 patients, found the BANA positive species, T. denticola, $P$. gingivalis, and B. forsythus to have the highest prevalence and to be present in the highest levels compared to over 40 other plaque species that were evaluated by DNA probes. ${ }^{25,26}$

When the BANA test was compared to DNA probes and immunological reagents that were highly specific for these organisms, it detected them in plaque samples with a level of accuracy that was comparable to the DNA probes and the immunological reagents; i.e. 89\% (BANA) vs $91.5 \%$ (DNA and antibody). The sensitivity of the BANA test for these bacteria in over 200 samples from 67 patients was $90 \%$ (BANA) compared to $92 \%$ for the DNA probes and immunological reagents. ${ }^{27}$

In a logistic regression model incorporating clinical parameters and the BANA test, the BANA test along with mobility, and level of attachment loss were significantly associated with the clinician's decision to recommend periodontal surgery as treatment for that particular tooth. ${ }^{28}$ 
However, it was not possible to determine presence/ absence and number of specific bacteria in red complex. Previous studies had indicated that presence of $P$. gingivalis and T. forsythia in subgingival plaque represented risk for attachment loss. N-benzyl-Dl-arginase-2-naphthalamide test is simple to use in clinical setting and the results can be obtained in a short time. So, the BANA test is suitable for this study. ${ }^{19,20}$

The mean red complex scores at the abutment teeth in contact with RPDs were significantly greater than for those at the nonabutment teeth $(\mathrm{p}<0.01)$. Furthermore, the regression analysis identified abutment teeth, PI and maintenance interval as significant independent predictors for positive scores of red complex $(\mathrm{p}<0.05)$. These results suggested that the microbiological risk for periodontitis of abutment teeth was greater than that for nonabutment teeth in RPD wearers, even if the PDindicates periodontal health. A previous study reported that the mean red complex score of teeth with periodontitis was 1.22 before treatment, which decreased to 0.52 at 90 days after surgical treatment. ${ }^{29}$

In the present study, the mean red complex score of nonabutment teeth (0.63) was similar to that reported after surgical treatment in the above study, indicating that the microbiological risk of nonabutment teeth examined in the present study was as low as teeth with healthy periodontium.

Clinical parameters are markers that help to describe disease or disease progression but they do not provide information on etiology. ${ }^{30}$ The present study assessed the presence of red complex as strongly associated periodontal pathogens that could be used as both markers and etiological agents of periodontal disease. It is possible that the wearing of RPDs increases the risk for periodontal disease of abutment teeth.

In our preliminary analysis, effect of RPD design on red complex and clinical parameters were not significant due to small sample size of open type RPDs. Thus, denture design was not included as predictors in the regression analysis.

The mean scores for PI at the abutment teeth were significantly higher than those for nonabutment teeth $(p=0.002)$. This finding is in agreement with previous studies, suggesting that wearing of RPD increases plaque accumulation at abutment teeth. Dental plaque functions as a biofilm that facilitates the attachment and aggression of microorganisms. ${ }^{31}$ Thus, it is reasonable that PI was identified as a significant predictor for positive score of red complex in the regression analysis.

Abutment teeth showed significantly higher mean scores for PI and GI compared with nonabutment teeth $(p<0.001)$. The coverage of marginal gingival tissue may be responsible for plaque accumulation and gingival inflammation of abutment teeth.

As the PD of examined teeth in this study was less than $4 \mathrm{~mm}$, it seemed that there was minimal alveolar bone loss.

Many investigators have recommended regular recalls for oral and denture maintenance, including professional care and reinforcement of plaque control, to preserve the periodontal health of abutment teeth in RPD wearers. ${ }^{8-10}$ In this study, maintenance interval longer than 6 months was the most critical factor among the significant predictors for presence of red complex $(\mathrm{p}<0.05)$. This result suggests that regular oral maintenance may reduce the microbiological risk for periodontitis of abutment teeth in RPD wearers. Decreasing the proportion of red complex species and the numbers of microorganisms in the subgingival area of teeth can be considered for infection control in periodontitis subjects. ${ }^{16}$

According to this concept, BANA hydrolysis test is simple method for detecting periodontal anaerobic bacterial infection, which is successfully used in clinical trials to evaluate changes in the subgingival microbiota after any type of treatment like RPDs and evaluate the risk for oral health.

\section{CONCLUSION}

The outcome of BANA test was highly significant in periodontally diseased abutment tooth of the removable partial denture. It can be used as a reliable indicator of BANA positive species in subgingival plaque for patients of removable partial denture. It is fast chair side kit to identify the red complex bacteria present in the abutment teeth of removable partial denture.

\section{REFERENCES}

1. Rosaiah K, Aruna K. The detection of BANA hydrolysis activity in chronic periodontitis. J Dent Sci 2013;5(1):44-49.

2. Bretz WA, Lopatin DE, Loesche WJ. Benzoyl-arginine naphthylamide (BANA) hydrolysis by Treponema denticoia and/or Bacteroides gingivalis in periodontal plaques. Oral Microbiol Immunol 1990;5(5):275-279.

3. LoescheWJ, BretzWA,KerschensteinerD,StollJA,SocranskySS, Hujoel PP, et al. Development of a diagnostic test for anaerobic periodontal infections based on Plaque Hydrolysis of Benzoyl-DL-Arginine-Naphthylamide. J Clin Microbiol 1990;28(7):1551-1559.

4. Bretz WA, Eklund SA, Radicchi R, Schork MA, Schork N, Schottenfeld $\mathrm{D}$, et al. The use of a rapid enzymatic assay in the field for the detection of infections associated with adult periodontitis. J Public Health Dent 1993;53(4):235-240.

5. Takaishi Y, Morri H, Miki T. The benzoyl-DL argininanaphthylamide (BANA) test and polymerase chain reaction measurement of pathogenic bacteria can assess the severity of periodontal disease. Int J Tissue React 2003;25(1):19-24. 
6. Alexandre de Andrade J, Feres M, et al. The ability of the BANA test to detect different levels of P. gingivalis, T. denticola and T. forsythia. Braz Oral Res 2010;24(2):224-230.

7. Schmidt EF, Bretz WA, et al. Correlation of the hydrolysis of benzoyl-arginine naphthylamide (BANA) by plaque with clinical parameters and subgingival levels of spirochetes in periodontal patients. J Dent Res 1988;67(12):1505-1509.

8. Carlsson GE, Hedegard B, Koivumaa KK. Studies in partial dental prosthesis. IV. Final results of a 4-year longitudinal investigation of dentogingivally supported partial dentures. Acta Odontol Scand 1965;23(5):443-472.

9. Derry A, Bertram U. A clinical survey of removable partial dentures after 2 years usage. Acta Odontol Scand 1970;28(5): 581-598.

10. Schwalm CA, Smith DE, Erickson JD. A clinical study of patients 1 to 2 years after placement of removable partial dentures. J Prosthet Dent 1977;38(4):380-391.

11. Ghamrawy EE. Qualitative changes in dental plaque formation related to removable partial dentures. J Oral Rehabil 1979;6(2):183-188.

12. Addy M, Bates JF. Plaque accumulation following the wearing of different types of removable partial dentures. J Oral Rehabil 1979;6(2):111-117.

13. Bissada NF, Ibrahim SI, Barsoum WM. Gingival response to various types of removable partial dentures. J Periodontol 1974;45(9):651-659.

14. Stipho HD, Murphy WM, Adams D. Effect of oral prostheses on plaque accumulation. Br Dent J 1978;145(2):47-50.

15. Faveri M, Gursky LC, Feres M, Shibli JA, Salvador SL, de Figueiredo LC. Scaling and root planing and chlorhexidine mouthrinses in the treatment of chronic periodontitis: a randomized, placebocontrolled clinical trial. J Clin Periodontol 2006;33(11):819-828.

16. Ximé nez-Fyvie LA, Haffajee AD, Socransky SS. Comparison of the microbiota of supra- and subgingival plaque in health and periodontitis. J Clin Periodontol 2000;27(9):648-657.

17. Loesche WJ, Bretz WA, Lopatin D, Stoll J, Rau CF, Hillenburg KL, et al. Multicenter clinical evaluation of a chairside method for detecting certain periodontopathic bacteria in periodontal disease. J Periodontol 1990;61(3): 189-196.

18. Silness J, Loe H. Periodontal disease in pregnancy. II. Correlation between oral hygiene and periodontal condition. Acta Odontol Scand 1964;22(1):121-135.
19. Silness J, Loe H. Periodontal disease in pregnancy. I. Prevalence and severity. Acta Odontol Scand 1963;21(6): 533-551.

20. Grossi S, et al. 'Assessment of risk of periodontal disease. I. risk indicators for attachment loss' J Periodontol 1994;65(3): 260-267.

21. Grossi SG, Genco RJ, Marthei EE, Ho AW, Kock G, Dunford A, Zambon JJ, Hausman E. Assessment of risk for periodontal disease. II Risk indicators for alveolar bone loss. J Periodontol 1995;66(1):23-29.

22. Laughon BE, Syed SA, Loesche WJ. API ZYM System for identification of Bacteroides spp. Caprocytophage, spp. and Spirochetes of oral origin. J Clin Microbiol 1982;15(1): 97-102.

23. Laughon BE, Syed SA, Loesche WJ. Rapid identification of Bacteroides gingivalis. J. Clin Microbiol 1982;15(2):345-346.

24. Tanner AC, Strempko, MN, Belsky, CA, McKinley EA. APIZYM and An-Ident reactions of fastidious Gram negative species. J Clin Microbiol 1985;22(3):353-355.

25. Haffajee AD, Cugini MA, Dibart S, Smith C, Kent RL Jr, Socransky SS. The effect of SRP on the clinical and microbiological parameters of periodontal disease. J Clin Periodontol 1997;24(5):324-334.

26. Socransky SS, Haffajee AD, Cuglini MA. Microbial complexes in subgingival plaque. J Dent Res 1997; 76(special issue): abst 302.

27. Loesche WJ, Lopatin DE, Giordano J, Alcoforado G, Hujoel P. Comparison of the benzoyl-DL-arginine-naphthylamide (BANA) test, DNA probes, and immunological reagents for ability to detect anaerobic periodontal infections due to Porphyromonas gingivalis, Treponema denticola, and Bacteroides forsythus. J. Clin Microbiol 1992;30(2):427-433.

28. Loesche WJ, Taylor GW, Giordano J, Hutchinson R, Rau CF, Chen Y-M, Schork MA. A logistic regression model for the decision to perform access surgery. J. Clin Periodontol 1997;24(3):171-179.

29. Neiva RF, Al-Shammari K, Nociti FH Jr, Soehren S, Wang HL. Effects of vitamin-B complex supplementation on periodontal wound healing. J Periodontol 2005;76(7):1084-1091.

30. BeckJD. Methods of assessing risk for periodontitis and developing multifactorial models. J Periodontol 1994;65(Suppl): 468-478.

31. Socransky SS, Haffajee AD. Dental biofilms: difficult therapeutic targets. Periodontol 2000, 2002;28(1):12-55. 